Original paper

\title{
Gafchromic film dosimetry: Four years experience using FilmQA Pro software and Epson flatbed scanners
}

\author{
M. Mathot*, S. Sobczak, M.-T. Hoornaert \\ Service de Radiothérapie, Centre Hospitalier Universitaire de Liège, Domaine Universitaire du Sart Tilman, B35, 4000 Liège, Belgium
}

\section{A R T I C L E I N F O}

\section{Article history:}

Received 3 December 2013

Received in revised form

21 May 2014

Accepted 23 June 2014

Available online 11 July 2014

\section{Keywords:}

GAFCHROMIC

Flatbed scanners

Multichannel correction

\begin{abstract}
A B S T R A C T
Purpose: s: To assess performance of FilmQA Pro software for pre-treatment patient-specific quality assurance (QA), using radiochromic films and two commercial flatbed scanners. To evaluate a novel multichannel approach compared to the classical red channel evaluation.

Material and methods: Patient films (mostly EBT2 films, one box of EBT3) were digitalized using successively two flatbed scanners: the A4-size Epson V750 and the A3-size Epson 10000XL. Prior to patient dose verification, basic characteristics of films and scanners were investigated. Patient films were analyzed using FilmQA Pro software, which enables to use the signal from all three colour channels (Red, Green, Blue).

Results: Compared to the red channel evaluation, multichannel evaluation presents better passing rates with regard to local gamma index. As expected, we obtained better results using A3-size scanner compared to A4-size scanner, especially when considering large region of interest. An observation of great interest was made for both scanners: after intensive use, a tilting in the blue transmittance profiles appeared in the lamp direction, making multichannel analysis unsuitable for accurate dose evaluation.
\end{abstract}

(C) 2014 Associazione Italiana di Fisica Medica. Published by Elsevier Ltd. All rights reserved.

\section{Introduction}

Radiochromic films are widely used for patient-specific dose verifications. They contain a sensitive continuous medium and offer very high spatial resolution. They do not present any volumetric averaging effect, as opposed to ionization chamber (IC) arrays. In contrast with diode and chamber arrays, they provide an isotropic response to radiation and they don't exhibit over-response to low energy photons owing to their near water equivalency. They can be irradiated in any position in any (water) phantom and they generate minimum perturbation of the beam fluence. Moreover, they are (almost) insensitive to visible light and do not require a well-controlled wet chemical processing in a dedicated darkroom facility.

Ashland Inc., Covington, KY, USA (formerly International Specialty Products) is the only supplier of radiochromic films (Gafchromic films) dedicated to therapeutic beams dose measurement. In the early days of 2009, the well-investigated EBT [1-5] was replaced by the model EBT2 [6-9]. By the end of 2011, a new EBT3 film was released $[10,11]$.

Many sources of uncertainties are involved in film dosimetry. Bouchard et al. [5] classified sources of uncertainties into five

\footnotetext{
* Corresponding author. Tel.: +32 43667940.

E-mail address: mmathot@chu.ulg.ac.be (M. Mathot).
}

categories: film manufacturing (film homogeneity), film manipulation (i.e. storage, cutting), film irradiation (phantom, setup), film digitalization (flatbed scanner), and film response characterization with absorbed dose (fitting calibration function). Richley et al. [7] stated that the overall uncertainty in EBT2 film dosimetry could be reduced from $3.8 \%$ to $1.5 \%$ by correcting for scanner nonuniformity. Arjomandy et al. [8] observed a $1.8 \%$ cumulative uncertainty arising from single EBT2 film non-uniformity, film-to-film variation and fitting uncertainty of the calibration data. Sorriaux et al. [11] emphasized a total uncertainty below $1 \%$ in the radiotherapy dose range ( $>1.5 \mathrm{~Gy}$ ) in photon mode using EBT3 films.

In this work we compared the "multichannel" analysis available in FilmQA Pro to the classical single (Red) channel method. We used successively two commercial flatbed scanners, the Epson V750 A4scanner and the manufacturer-recommended Epson 10000XL A3scanner. Prior to patient evaluation, some basic characteristics of both scanners and film types were investigated.

\section{Materials and methods}

\section{Radiochromic film and scanner models used}

The structure of EBT2 and EBT3 films is described in Refs. [10,12]. The same active component is used and the nominal thickness of 
the active layer is the same. The difference between film versions is twofold. First, EBT3 film has a symmetric structure, eliminating concerns about which film side should face the scanner glass. Secondly, in EBT3 film the substrates embed microscopic silica particles in the outer surface, frustrating the formation of Newton rings. Owing to their atomic composition, EBT2 and EBT3 films have near-tissue-equivalent density $\left(\mathrm{Z}_{\mathrm{eff}}^{\mathrm{EBT2}}=6.84\right.$ compared to $\mathrm{Z}_{\text {eff }}^{\text {Water }}=7.3$ ). Radiochromic films are better stored in a dry and dark environment. Girard et al. [13] reported a maximum rate of optical density change of $\pm 0.15 \%$ per ${ }^{\circ} \mathrm{C}$ in the red channel. They also showed that relative humidity variations ranging from $80 \%$ to $20 \%$ could introduce dose errors of up to $15 \%$. We pre-cut films one day prior to irradiation to allow for disturbances around the edges to mechanically relax. We do not use gloves and we are careful not to touch film area where evaluation will be performed. From end 2008 to mid-2013, we irradiated 1225 EBT2 film sheets (49 boxes) from 12 different lots. We used only one box of EBT3 film.

Initially we have been using an Epson A4-size V750 Pro scanner (Seiko Epson Corporation, Nagano, Japan), then we moved to an A3size Epson 10000 XL. A technical description can be found in Ref. [7]. The scanner is used in transmission mode and film images are acquired in 48 bit red-green-blue (RGB) mode at a resolution of $72 \mathrm{dpi}(0.35 \mathrm{~mm}$ pixel size). In each channel, the maximum value of the analogue-to-digital converter (ADC) scale is $2^{16}-1(65,535)$. These raw image ADC data are hereafter referred to as "pixel values" $(\mathrm{PV})$, ranging from 0 (no signal, zero transmission) to 65,535 (maximum signal, total transmission). We use the professional mode with all image adjustments (such as contrast enhancement) and colour corrections turned off.

\section{Analysis software}

Since 2009 we have been using FilmQA Pro software (Ashland Inc), a quantitative analysis tool designed for film scanning, PV extraction, PV conversion to dose, and comprehensive dose analysis. A feature of the software is the ability to use pixel values from all colour channels together in dose map construction, providing "corrected" red, green and blue dose maps for further analysis. This evaluation method is called "triple channel evaluation", or "multichannel evaluation". According to Micke et al. [14], it allows for the separation of the dose-dependent part of the signal from the dose independent-part, the latter stemming from a variety of disturbances related to film manufacturing (structure heterogeneities) as well as scanner artefacts (i.e. noise) or film manipulation (i.e. dust, fingerprints). Practically, the method varies the dose values until the corresponding pixel values are best matched for all three colour channels. As dose values for the different corrected channels should be in close agreement, significant offsets indicate a mismatch between the scanned film and the calibration data. These offsets are displayed in so-called "consistency maps". Clear offsets would occur, for example, when orientations of calibration films and patient films are not the same.

\section{Film irradiation}

Film irradiation was performed on two Elekta Synergy S (Elekta $A B$, Stockholm, Sweden) linacs equipped with Beam Modulator collimator. Step-and-shoot IMRT (SS-IMRT) and VMAT treatments were delivered through $6 \mathrm{MV}$ photon beams exclusively. We used DMPO and SmartArc modules in Pinnacle v 9.0. (Philips Medical Systems, The Netherlands). The OmniPro-I'mRT phantom (IBA Dosimetry AB, Uppsala, Sweden), a modular phantom consisting of a universal body shaped section made of RW3 material (1.045 g/ $\mathrm{cm}^{3}$ ) can accommodate pre-cut film pieces of $16 \times 16 \mathrm{~cm}^{2}$ between slabs in a coronal plane. In addition to film evaluation, we performed ionization chamber measurements with a crosscalibrated CC13 chamber (IBA Dosimetry) fitted at the phantom geometrical centre. One single film per patient, positioned $2 \mathrm{~cm}$ above the isocentre plane, was used. Prior to patient film irradiation, a "control film" was exposed to an open $10-\mathrm{cm}$ square field (225 cGy at the film location).

\section{Film scanning}

The reproducibility of film positioning is a key point due to the non-uniform scanner response over the scan field. It has been shown [2,7] that raw pixel values obtained from any scanner readout are dependent on both the received dose and the film pixel position on the scanner glass, especially in the transverse direction (the direction perpendicular to the scan direction, or parallel to the scanner lamp). This well-known artefact, referred to as "lateral effect", causes transmission PV to decrease as the lateral distance from the scan axis increases. This effect increases at high doses. In this study a template is fitted on the scanner glass so that film pieces are immobilized in the central area of the scanner bed, in a reproducible way.

Due to the anisotropic light scattering in radiochromic films, film orientation must be kept constant. Film orientation is referred to as "portrait" when the long axis of the film is parallel to the scan direction. The orientation perpendicular to the portrait orientation is referred to as "landscape" orientation. According to the manufacturer's recommendations, all films were scanned in landscape orientation in this study.

In Ref. [8], Arjomandy et al. recommend considering the average of multiple readings of the same film, in order to minimize lamp warm-up dependence and to improve the signal to noise ratio. As proposed by Saur et al. [3], we scanned every film twice and considered only the second reading for further analysis. Prior to scanning session, five successive blank scans are taken for scanner warm-up.

Regarding post exposure polymerization, we used a time period of about $15 \mathrm{~h}$ between film exposure and film scanning, within a time window of $2 \mathrm{~h}[10]$.

\section{Treatment verification using an independent electronic device}

The Delta4 (ScandiDos AB, Uppsala, Sweden) electronic device was used for patient-specific QA, in order to evaluate gamma passing rates with another independent system. The Delta4 was calibrated according to manufacturer's recommendations, against a Farmer type ionization chamber. The device was always positioned at the machine isocentre (no longitudinal shift). Gamma evaluation was performed in absolute mode.

\section{Experiments}

\section{Scanner warm up and reproducibility}

For both scanners, we quantified scanner warm up effects at two dose levels (20 cGy and $300 \mathrm{cGy}$ ). We scanned EBT2 film pieces, batch A08171101A, in the central area of the scanner. The region of interest (ROI) has a dimension of 1 in. $\times 1$ in. (72 pixels $\times 72$ pixels). Without any preview, we measured ten times consecutively the mean pixel values in the ROI, in the three channels. To assess scanner reproducibility at various dose levels, we scanned eight times all film pieces used for calibration (from $10 \mathrm{cGy}$ to $360 \mathrm{cGy}$ ), after proper warm up of the lamp, for both scanners. Following this experiment, scanner reproducibility was assessed on regular basis (more or less quarterly) by scanning eight times the control films exposed to a uniform dose (225 cGy). 
Intra-batch homogeneity and intra-sheet uniformity

A frequently highlighted issue in early batches of EBT2 concerned local heterogeneities due to manufacturing inconsistency, leading to uncertainties in dose determination far beyond commonly accepted tolerance levels [6]. We assessed intra-batch homogeneity at zero dose only, scanning five EBT2 films in the central area of both scanners and five EBT3 films using Epson 10000XL. To study intra-film homogeneity, EBT2 and EBT3 film sheets were randomly selected and cut into eight pieces. In order to mitigate influence of scanner reproducibility, results were averaged over three readings. Finally we compared, for EBT2 films only, intrafilm homogeneity at $0 \mathrm{cGy}$ and $225 \mathrm{cGy}$.

\section{Film calibration}

Eight pieces out of a single film sheet were exposed to increasing dose levels (i.e. 10 cGy, 30 cGy, 60 cGy, 100 cGy, 150 cGy, $210 \mathrm{cGy}, 280 \mathrm{cGy}$ and $360 \mathrm{cGy})$ under reference conditions $(100 \mathrm{~cm}$ $\mathrm{SAD}, 10 \mathrm{~cm}$ depth in solid water, $10 \mathrm{~cm}^{2}$ field size). Using FilmQA Pro, the average PV is measured for all channels in a central $72 \times 72$ square pixel ROI, and a calibration function is fitted onto the calibration data points. In FilmQA Pro a variety of monotonic functions is provided. As pointed out in Micke et al. [14], polynomial functions are not recommended. FilmQA Pro provides users with invertible functions whose extrapolated values asymptote to constants, like the function "colour reciprocal linear vs dose", in Eq. (1).

$\mathrm{PV}(D)=a+\frac{b}{D-c}$

where PV and $D$ are pixel value and dose, respectively.

\section{Application to patient specific QA}

Absolute dose measurements were compared to Pinnacle calculations, using the collapsed cone convolution/superposition (CCC) algorithm and a $1-\mathrm{mm}$ resolution in the coronal plane located at the film position. A noise filter was applied onto dose maps generated in FilmQA Pro, since residual noise may lead to an overestimation of gamma passing rates.

Head-and-neck patients are treated with simultaneously integrated boost (SIB), typically 54-60-70 Gy in 30 fractions. For prostate patients, hypofractionation results in prescribed dose up to $2.63 \mathrm{~Gy}$ per fraction. Our results are reported in terms of global gamma index with $5 \mathrm{cGy}$ dose difference (DD)/3 mm distance-toagreement (DTA) criteria. We decided to stick to this absolute dose difference criterion since a percentage of maximum dose would overestimate passing rates for integrated boost treatments. We also perform a local gamma evaluation using 3\% local dose difference/3 mm DTA criteria, with an inferior threshold of $20 \mathrm{cGy}$. Gamma analysis is manually restricted to a rectangular area encompassing the apparent dose-induced darkening zone. The ROI is positioned at least $5 \mathrm{~mm}$ from the film edges. This means that for large PTV, the gamma index evaluation is performed over a $15 \times 15 \mathrm{~cm}^{2}$ area. In this study the "corrected red" dose map is the only one we consider in multichannel evaluation.

Student $t$ test was used for comparative analysis. Differences are considered statistically significant when $p<0.05$.

\section{Results}

Scanner warm up. Scanner reproducibility

Scanner warm up effects are negligible, except in the blue channel at low dose using the Epson V750. In this case, normalized
PV increased from 43.20 to 43.76 during warm up of the lamp (a normalized PV of 100 indicates full transmission, i.e. raw PV of $65,535)$. In the red and green channels, the relative standard deviations of the ROI mean PV during warm up are below $0.1 \%$, for both scanners.

Regarding scanner reproducibility after proper warm up, we obtained for both scanners standard deviations below $0.25 \%$ in the blue channel and below $0.15 \%$ in the red and green channels. Using control films, we observed no drift over the lifetime of the scanners.

\section{Intra-batch/intra-film homogeneity}

To illustrate intra-batch homogeneity at zero dose, the PV standard deviations between films from the same batch are shown in Fig. 1.

As Epson V750 and Epson 10000XL present similar reproducibility, Fig. 1 shows that intra-batch homogeneity is better for the most recent EBT2 batch (A08171101A), confirming the improvements claimed by the manufacturer. The measured batch homogeneity (averaged over three readings) lies within the range of scanner reproducibility.

Intra-film uniformity at zero dose is illustrated in Fig. 2. EBT3 (batch A03051205) uniformity is markedly degraded compared to EBT2 films.

Figures 1 and 2 show that intra-film uniformity at zero dose for EBT3 is poorer than intra-batch homogeneity measured in the central region of film sheets. From that we conclude that manufacturing heterogeneities are prominent in the peripheral parts of film sheets for this batch.

Comparing EBT2 (batch A09271204) intra-film homogeneity at 0 cGy and 225 cGy, we observed that relative standard deviations were slightly lower at high dose. From this single experiment, we assumed that film homogeneity is not degraded in clinical dose range, compared to results in Figs 1 and 2.

\section{Film calibration}

In Fig. 3 are shown calibration functions of the type "colour reciprocal linear vs dose", see Eq. (1).

The first time we used EBT3 films, none of the fitting functions available in FilmQA Pro (i.e. color reciprocal linear vs dose, rational linear (quadratic, cubic) vs dose, and exponential universal) was able to fit the calibration data in the blue channel. Using another EBT3 film sheet, a new calibration was done and resulted in a good fit. We never observed this problem with EBT2 films, whatever the batch number. Further investigation, using more recent EBT3 film batches, is mandatory to draw any conclusion.

\section{Patient specific QA}

In Table 1 are shown passing rates for prostate (no lymph node included) and head-and-neck patients, using landscape orientation and Epson V750.

For head-and-neck patients more evaluation points failed the gamma criteria. According to unpaired $t$ test, differences between film batches are not significant ( $p=0.764$ for prostate, $p=0.092$ for head-and-neck).

After two years of intensive use of the Epson V750 scanner (i.e. around 5000 scans, including warm-up and experiments), we observed a slight slope in raw PV profiles on the control film pieces, in the lamp direction. This slope suddenly appeared and was prominent in the blue channel. Consequently we accelerated the purchase of an A3-size Epson 10000XL scanner. This unwanted behaviour of the scanner is discussed in the Discussion Section. 


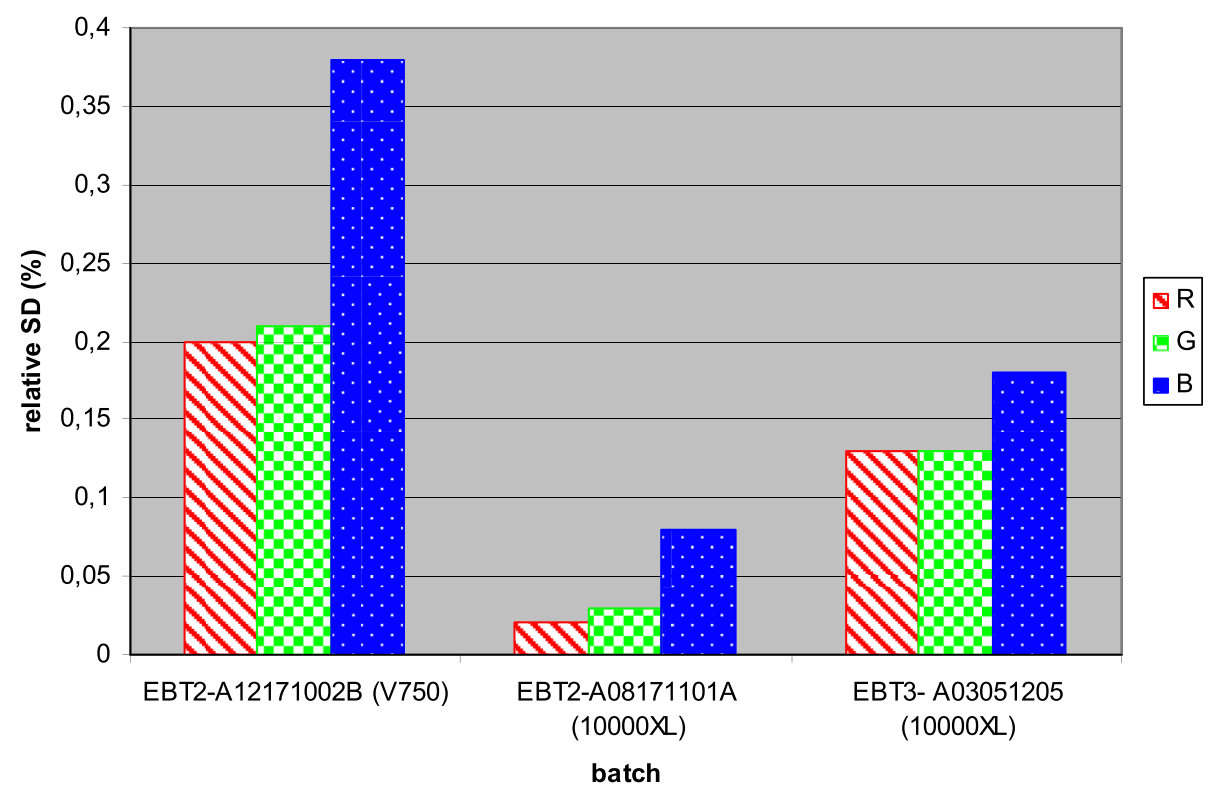

Figure 1. Intra-batch homogeneity of EBT2 and EBT3 films, using both scanners.

Gamma passing rates using Epson $10000 \mathrm{XL}$ in landscape orientation are shown in Table 2. Single channel evaluation is compared to multichannel (corrected red) correction algorithm. We also compared global gamma index ( $5 \mathrm{cGy} / 3 \mathrm{~mm}$ criteria) to local gamma index $(3 \% / 3 \mathrm{~mm})$. All localized prostate patients underwent VMAT treatments, while 35 out of 81 head-and-neck patients received step-and-shoot treatment, as well as 7 out of 16 pelvis patients.
Regarding global passing rates, results are very similar between single channel and multichannel evaluation. Using a paired $t$ test, we obtain two-tailed $p$ values equal to $0.801,0.372$ and 0.615 for respectively prostate, pelvis and head and neck patients. On the other hand, multichannel evaluation presents better passing rates with regard to local gamma index, with significant $p$ values ( $p=0.016,0.049$ and 0.028 for prostate, pelvis and head and neck patients, respectively). Local index criterion is more stringent than

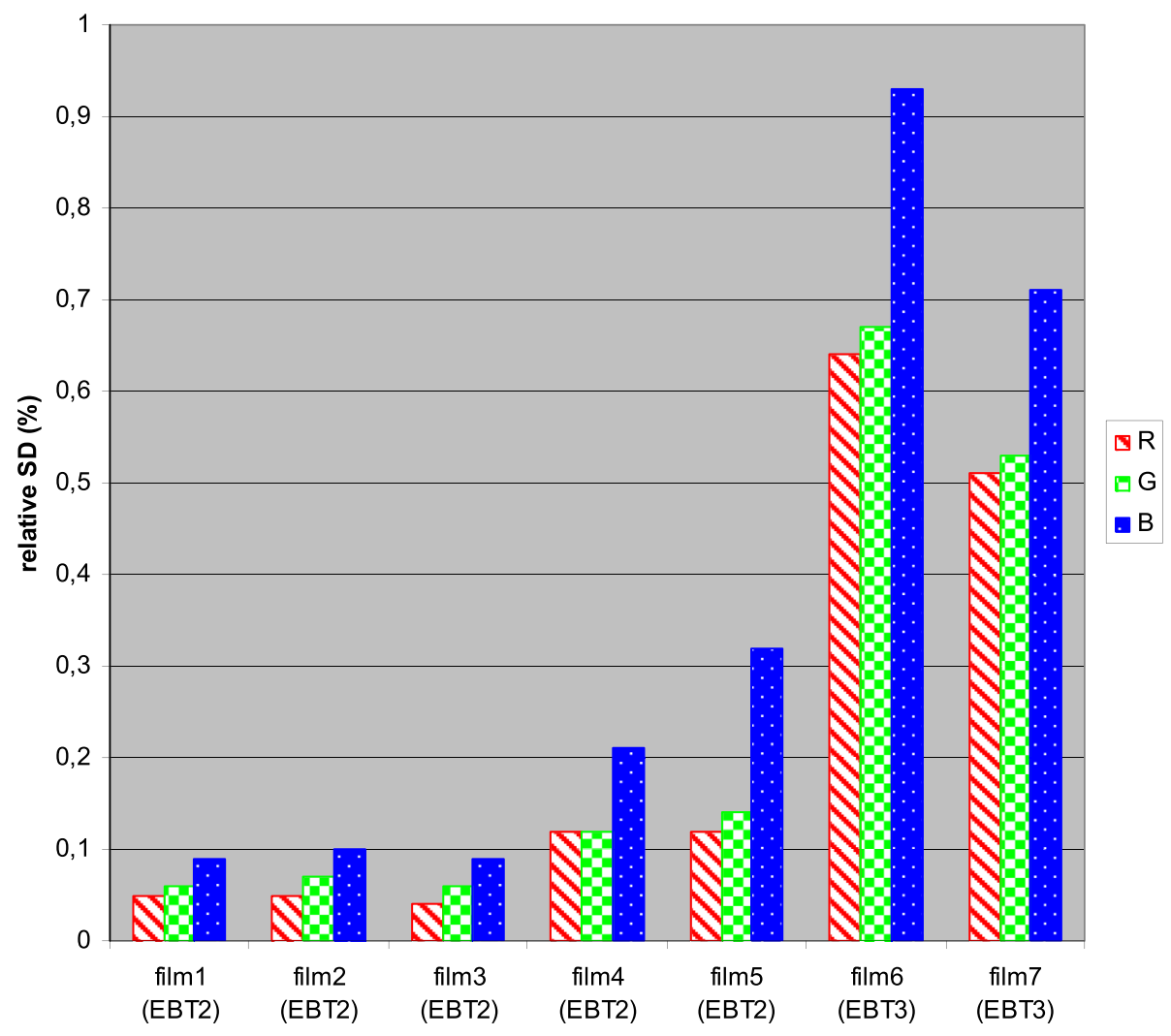

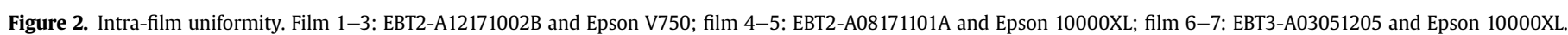




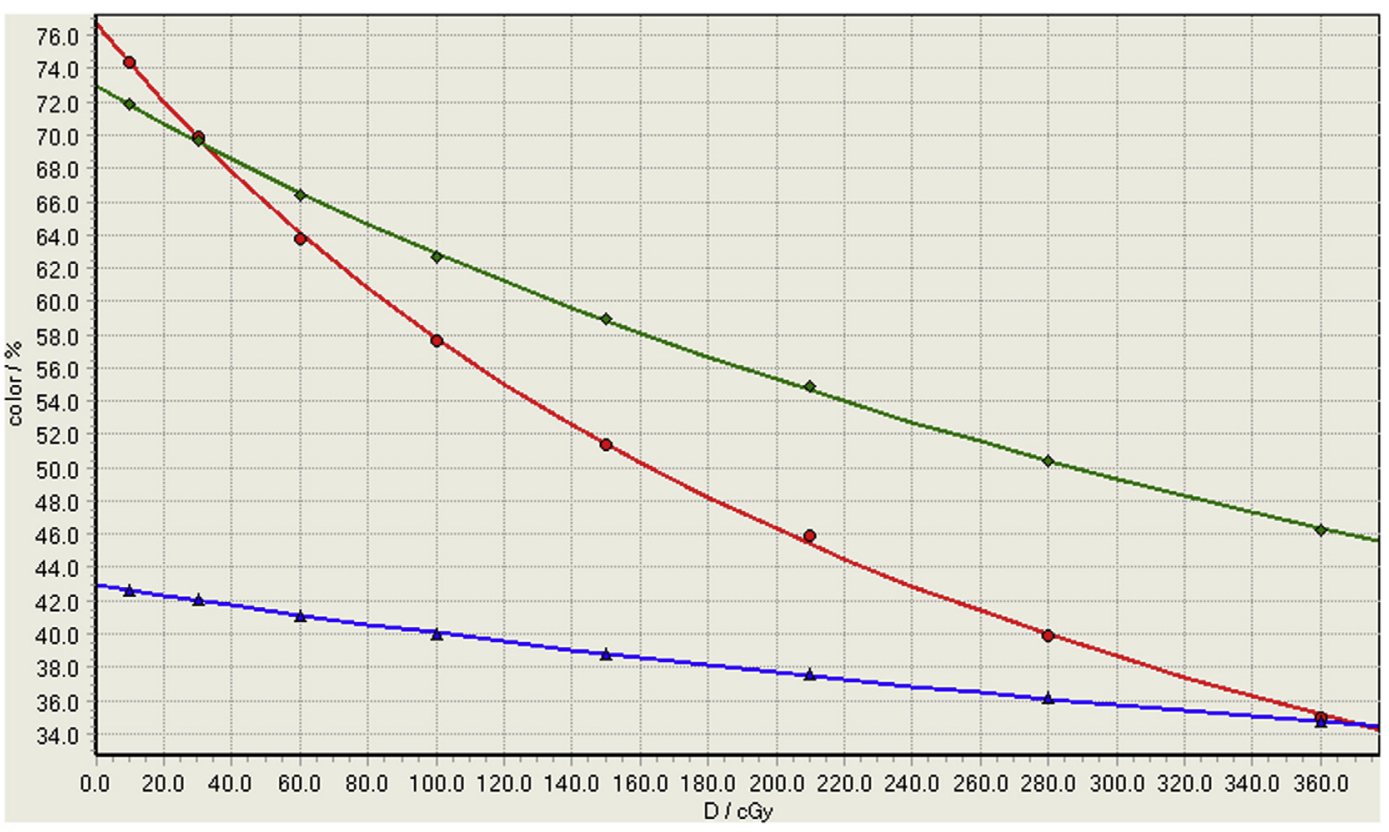

Figure 3. Calibration functions of type "color reciprocal linear vs dose”. EBT2-A12171002B. Normalized PV (\%) are plotted against dose (cGy).

global index in "low" dose regions (in regions receiving less than 167 cGy). When considering VMAT and step-and-shoot patients separately, no significant difference appears (unpaired $t$ test), either for pelvis or head-and-neck patients. Average passing rates are very close whatever the gamma criterion or the evaluation algorithm, with no advantage for step-and-shoot versus VMAT.

As we used one single box of EBT3 films, results are not shown (thirteen patients only). Passing rates are slightly lower than those displayed in Table 2.

When using multichannel approach we systematically verified the consistency between the three colour channels. We observed arithmetic consistency (i.e. average difference between corrected doses in the three channels) between $1.5 \mathrm{cGy}$ and $2.5 \mathrm{cGy}$, which is consistent with expected values [14].

After one year of scanning with Epson 10000XL (around 3000 scans), we encountered the same problem we observed with Epson V750. Pixel value profiles in the lamp direction presented a gradual tilting, resulting in a slope of many percents in dose using multichannel approach. In Fig. 4 are shown PV profiles in the lamp direction (a) and corresponding corrected dose profiles (b), for a control film exposed to uniform radiation (225 cGy).

\section{Discussion}

Using the control films irradiated with a square open field prior to patient measurement, progressive dysfunctions with both Epson scanners were discovered (see Fig. 4 for Epson 10000XL). After one (Epson 10000XL) or two (Epson V750) year(s) of use, a gradual tilting of PV profiles in the lamp direction was observed. This tilting

Table 1

Gamma passing rates for prostate (54 patients) and head-and-neck (43 patients), using two EBT2 film batches. Films scanned in landscape orientation with Epson V750 and evaluated through multichannel correction algorithm. Agreement thresholds are $5 \mathrm{cGy}-3 \mathrm{~mm}$.

\begin{tabular}{lllll}
\hline & Prostate & H\&N & Prostate & H\&N \\
& A09031001B & A09031001B & A12171002B & A12171002B \\
\hline Pass rate (\%) & $95.1 \pm 3.9$ & $88.1 \pm 7.1$ & $95.4 \pm 3.5$ & $92.44 \pm 6.1$ \\
\hline
\end{tabular}

is prominent in the blue channel, with normalized raw PV rising from $36.5 \%$ to $37.5 \%$ over the flat area of a $10-\mathrm{cm}$ open field, see Fig. 4 (a). In the green and red (in web version) channels, there is no significant tilting, with normalized PV within the range of measurement uncertainty. The corrected dose maps in multichannel analysis are significantly impacted, despite the lower weight of the blue channel in the algorithm. This lower weight of the blue channel is a consequence of the shallow slope of the calibration function in the blue channel, compared to the red and green (in web version) curves (see Fig. 3 and Ref. [14]). Anyway the magnification of the slope in terms of dose is mainly due to the slope of the calibration functions, more sensitive in relative dose than in relative PV.

This problem appeared suddenly, reinforced over time (during a few weeks time) and then stabilized, for both scanners. Regrettably consistency maps are unable to detect the problem, since dose values between corrected channels remain very close.

Epson technical support (epson.fr) was not able to help us, arguing that "their document scanners are not officially designed for irradiated films digitalization". The tilting is not visible in reflection mode (reflection mode was used by Butson et al. in Ref. [15], with good results), where the lamp is below the scanner bed. In transmission mode, the working lamp is in the scanner lid (optional transparency unit). Based on this, we think that the tilting is mainly due to the transmission lamp. To verify these conclusions, we plan to order a new transparency unit. To the best of our knowledge, this undesirable behaviour has not been reported so far

Table 2

Comparison of gamma passing rates for prostate (31 patients), pelvic (16 patients) and head-and-neck patients (81 patients). Gamma criteria are $5 \mathrm{cGy}-3 \mathrm{~mm}$ (global) and $3 \%-3 \mathrm{~mm}$ (local). EBT2 films are scanned using Epson 10000XL in landscape orientation. Batch numbers are A08171101A, F12170901A and A03221201.

\begin{tabular}{llllll}
\hline Pass rates (\%) & \multicolumn{2}{l}{ Red channel } & & \multicolumn{2}{l}{ Multichannel } \\
\cline { 2 - 3 } & Global & Local & & Global & Local \\
\hline Prostate only & $97.0 \pm 2.9$ & $93.1 \pm 3.7$ & & $97.0 \pm 2.6$ & $94.3 \pm 2.7$ \\
Pelvis & $95.8 \pm 3.0$ & $95.0 \pm 3.3$ & & $95.1 \pm 4.4$ & $96.0 \pm 3.0$ \\
Head and neck & $97.0 \pm 3.5$ & $94.0 \pm 5.6$ & & $97.2 \pm 3.0$ & $95.2 \pm 3.7$ \\
\hline
\end{tabular}




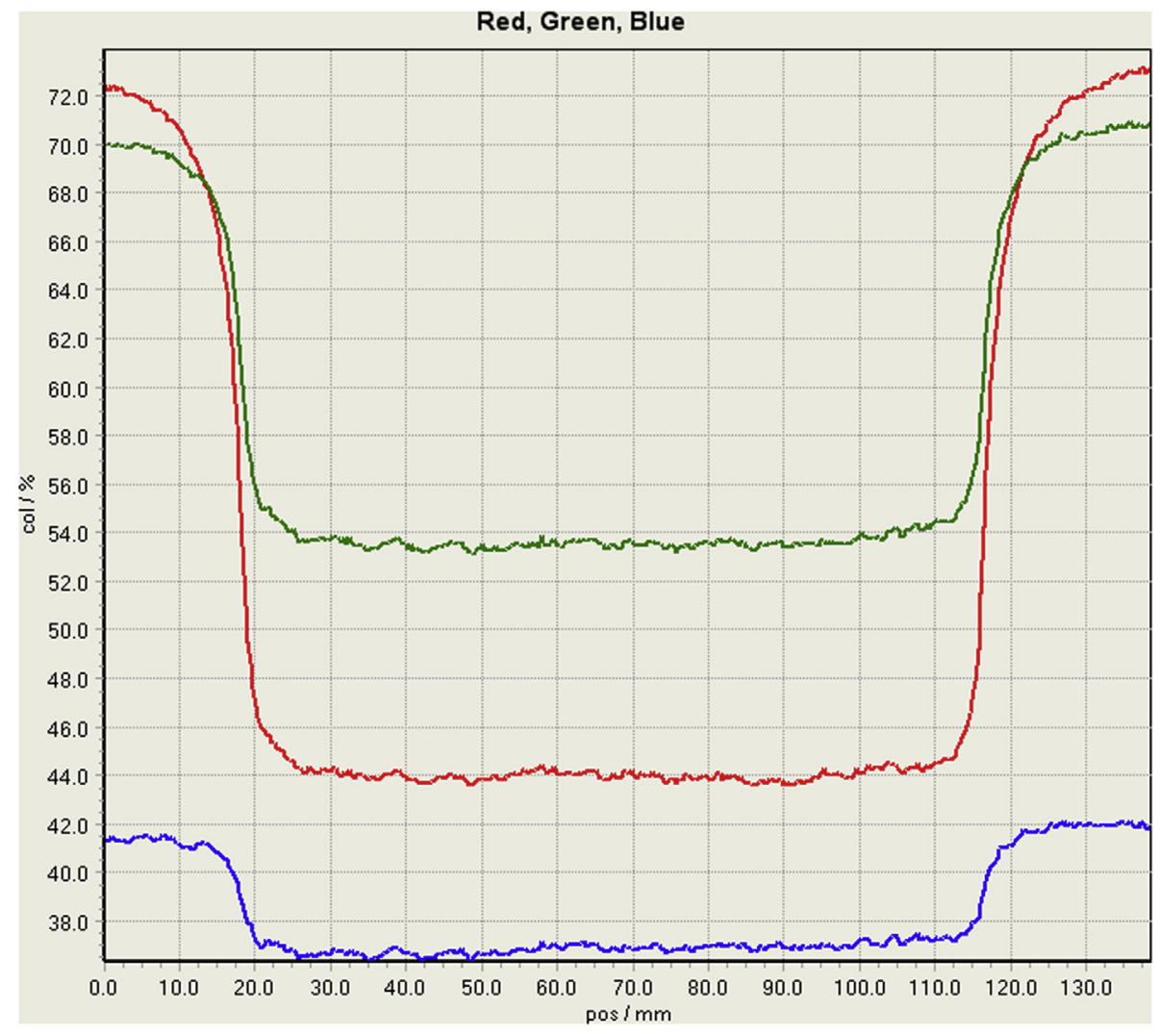

(a)

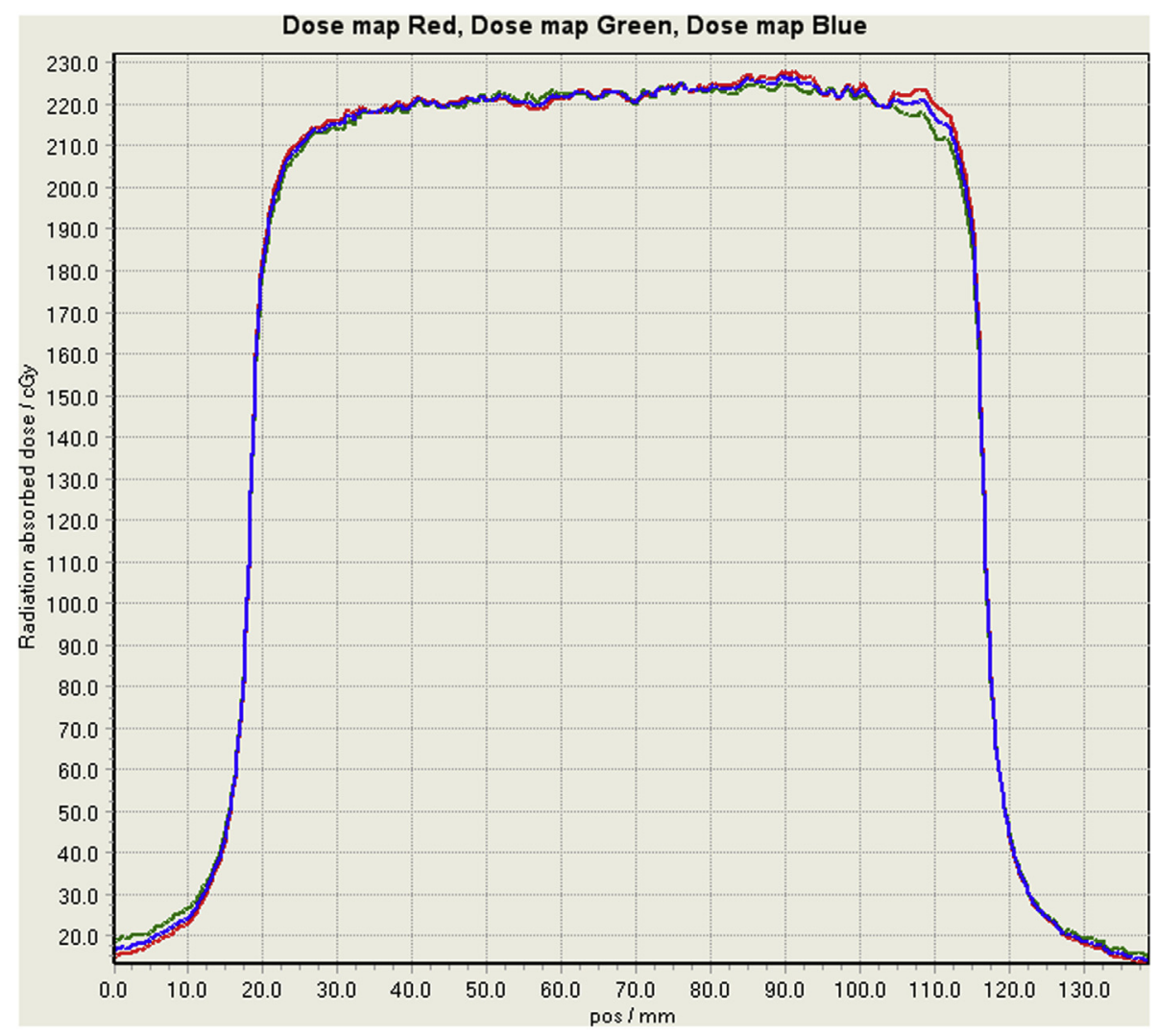

(b)

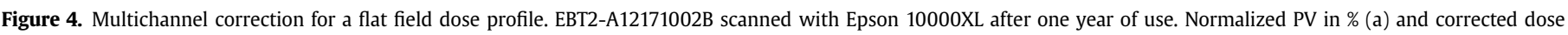
profiles in cGy (b) in the lamp direction. 
for commercial flatbed scanners dedicated to Gafchromic film analysis.

As regards intra-sheet and intra-batch homogeneity, we recommend the film batch to be examined (at zero dose) before clinical use, especially when early batches are considered.

Using the A4-size Epson V750, we obtained good passing rates for prostate patients, for whom the region of interest is (roughly) less than $10 \mathrm{~cm}$. More discrepancies were observed for head-andneck patients (Table 1 ), due to the scanner lateral distortions. Using the A3-size Epson 10000XL (Table 2), gamma passing rates were improved for head-and-neck patients, due to the better spatial uniformity in the central area of the scanner bed [4].

Our comparative results between single channel evaluation and triple channel method in FilmQA Pro confirm a noticeable gain to the benefit of the latter, in terms of gamma passing rates. The comparison between TPS calculations and measurements was also based on a cross-calibrated CC13 IC (together with film measurements) and the Delta4. All patients considered, CC13 measurements showed a slight underdosing $(-1.2 \% \pm 0.7 \%$ ( $1 \mathrm{SD}))$. Using Delta4, local gamma passing rates $(3 \% / 3 \mathrm{~mm})$ were $97.9 \% \pm 1.7 \%$, step-and-shoot treatments showing passing rates slightly higher than VMAT. The $10-\mathrm{cm}$ square beam irradiation (see Ref. [16] for multichannel evaluation in standard configuration), showed perfect agreement (100\% passing rates) using control films and Delta4, except at field edges from time to time. Based on these results, we gained confidence in TPS accuracy and (dynamic) beam delivery. Higher local gamma passing rates obtained using multichannel algorithm (closer to Delta4 results) suggest that some film/scanner artefacts are effectively mitigated compared to single channel analysis.

Using one box of EBT3, we encountered difficulties we never observed using many batches of EBT2, i.e. fitting problems during calibration and poorer intra-film uniformity. Based on this limited experience, we decided to stick to EBT2 films for the future, keeping in mind that similar dosimetric performance between EBT2 and EBT3 was described in thorough publications $[10,11]$.

In this study, no attempt was made to correct for the welldocumented dose-dependent non-uniform spatial response of flatbed scanners. Recent publications proposed elegant solutions to this limitation, for example Girard et al. [13] and Crijns et al. [17]. These kinds of investigations are outside the scope of this work, since our purpose is to report about our experience with commercially available software and "plug and play" solution.

In addition to scanner reproducibility and film homogeneity, many uncertainties are involved in film dosimetry. Interesting discussion and analysis can be found in Refs. [7,18,19]. According to [7,19], a global relative uncertainty of less than $3 \%$ can be achieved in the central area of the scanner bed using our methodology.

Finally, physicists interested in starting film dosimetry should be aware of the time investment required. Film preparation (when cutting is needed), film setup (for every patient), film scanning, film spatial registration and calibration of new batches are time consuming. With no attempt to speed up the process (as in Ref. [12]), the time needed per patient is around $30 \mathrm{~min}$, to be compared to the 10 min needed using dedicated electronic devices offering online readout and evaluation.

\section{Conclusion}

Radiochromic films offer many unique features. There is no other affordable detector providing sub-millimetre spatial resolution, no angular dependence and weak energy dependence. Based on our experience, we can state that Gafchromic films are reliable for verification of highly-modulated dose distributions. The film supplier has developed brand new software that streamlines and optimizes PV conversion into dose and provides users with valuable warnings (consistency maps) for existing errors.

Nevertheless, in order to ensure sufficient accuracy, extreme caution is necessary when manipulating, irradiating and scanning films. Special attention should be given to the behaviour of the scanner, since film digitalization is by far the most critical step. Commercial flatbed scanners are not designed for film dosimetry and have to be used with great caution (fixed location, stable environmental conditions, informed users). It is mandatory to monitor their performances on regular basis, to avoid major discomfiture. To our knowledge, this study is the first to highlight stochastic ageing artefacts for flatbed scanners.

\section{Acknowledgements}

The authors would like to thank Andre Micke and David Lewis of Ashland, Inc., for their availability and helpful discussions.

\section{References}

[1] van Battum LJ, Hoffmans D, Piersma H, Heukelom S. Accurate dosimetry with Gafchromic EBT film of a 6 MV photon beam in water: what level is achievable? Med Phys 2008;35(2):704-16.

[2] Menegotti L, Delana A, Martignano A. Radiochromic film dosimetry with flatbed scanners: a fast and accurate method for dose calibration and uniformity correction with single film exposure. Med Phys 2008;35(7): $3078-101$.

[3] Saur S, Frengen J. Gafchromic EBT film dosimetry with flatbed CCD scanner: a novel background correction method and full dose uncertainty analysis. Med Phys 2008;35(7):3094-101.

[4] Ferreira BC, Lopes MC, Capela M. Evaluation of an Epson flatbed scanner to read Gafchromic EBT films for radiation dosimetry. Phys Med Biol 2009;54(4): 1073-85.

[5] Bouchard H, Lacroix F, Beaudoin G, Carrier JF, Kawrakow I. On the characterization and uncertainty analysis of radiochromic film dosimetry. Med Phys 2009;36(6):1931-46.

[6] Hartmann B, Martišíková M, Jäkel O. Technical note: homogeneity of Gafchromic EBT2 film. Med Phys 2010;37(4):1753-6.

[7] Richley L, John AC, Coomber H, Fletcher S. Evaluation and optimization of the new EBT2 radiochromic film dosimetry system for patient dose verification in radiotherapy. Phys Med Biol 2010;55(9):2601-17.

[8] Arjomandy B, Tailor R, Anand A. Energy dependence and dose response of Gafchromic EBT2 film over a wide range of photon, electron, and proton beam energies. Med Phys 2010;37(5):1942-7.

[9] Carrasco MA, Perucha M, Luis FJ, Baeza M, Herrador M. A comparison between radiochromic EBT2 film model and its predecessor EBT film model. Phys Med 2013;29:412-22.

[10] Reinhardt S, Hillbrand M, Wilkens JJ, Assmann W. Comparison of Gafchromic EBT2 and EBT3 films for clinical photon and proton beams. Med Phys 2012;39(8):5257-62.

[11] Sorriaux J, Kacperek A, Rossomme S, et al. Evaluation of Gafchromic EBT3 films characteristics in therapy photon, electron and proton beams. Phys Med 2013:29:599-606.

[12] Lewis D, Micke A, Yu X, Chan MF. An efficient protocol for radiochromic film dosimetry combining calibration and measurement in a single scan. Med Phys 2012;39(10):6339-50.

[13] Girard, et al. Reference dosimetry using radiochromic film. J Appl Clin Med Phys 2012;13(6):339-53.

[14] Micke A, Lewis DF, Yu X. Multichannel film dosimetry with nonuniformity correction. Med Phys 2011:38(5):2523-34.

[15] Butson, et al. Energy response of the new EBT2 radiochromic film to x-ray radiation. Radiat Meas 2010;45:836-9.

[16] van Hoof SJ, Granton PV, Landry G, Podesta M, Verhaegen F. Evaluation of a novel triple-channel radiochromic film analysis procedure using EBT2. Phys Med Biol 2012;57(13):4353-68.

[17] Crijns W, Maes F, van der Heide UA, Van den Heuvel F. Calibrating page sized Gafchromic EBT3 films. Med Phys 2013:40(1). 012102-1-012102-13.

[18] Devic S. Radiochromic film dosimetry: past, present and future. Phys Med $2011 ; 27: 122-34$

[19] Nakano M, Hill R, Whitaker M, Kim J, Kuncic Z. A study of surface dosimetry for breast cancer radiotherapy treatments using Gafchromic EBT2 film. J Appl Clin Med Phys 2012;13(3):83-97. 